ARTICLE

DOI: $10.1038 / s 41467-018-03270-4$

\title{
Evidence that DNA polymerase $\delta$ contributes to initiating leading strand DNA replication in Saccharomyces cerevisiae
}

\author{
Marta A. Garbacz ${ }^{1}$, Scott A. Lujan (10 ${ }^{1}$, Adam B. Burkholder ${ }^{2}$, Phillip B. Cox ${ }^{1}$, Qiuqin Wu ${ }^{3}$, Zhi-Xiong Zhou ${ }^{1}$ \\ James E. Haber ${ }^{3}$ \& Thomas A. Kunkel ${ }^{1}$
}

To investigate nuclear DNA replication enzymology in vivo, we have studied Saccharomyces cerevisiae strains containing a pol2-16 mutation that inactivates the catalytic activities of DNA polymerase $\varepsilon$ (Pol $\varepsilon$ ). Although pol2-16 mutants survive, they present very tiny spore colonies, increased doubling time, larger than normal cells, aberrant nuclei, and rapid acquisition of suppressor mutations. These phenotypes reveal a severe growth defect that is distinct from that of strains that lack only Pol $\varepsilon$ proofreading (pol2-4), consistent with the idea that Pol $\varepsilon$ is the major leading-strand polymerase used for unstressed DNA replication. Ribonucleotides are incorporated into the pol2-16 genome in patterns consistent with leading-strand replication by $\mathrm{Pol} \delta$ when Pol $\varepsilon$ is absent. More importantly, ribonucleotide distributions at replication origins suggest that in strains encoding all three replicases, Pol $\delta$ contributes to initiation of leading-strand replication. We describe two possible models.

\footnotetext{
${ }^{1}$ Genome Integrity and Structural Biology Laboratory, National Institute of Environmental Health Sciences, NIH, DHHS, Research Triangle Park, Durham, NC 27709, USA. ${ }^{2}$ Integrative Bioinformatics Support Group, National Institute of Environmental Health Sciences, NIH, DHHS, Research Triangle Park, Durham, NC 27709, USA. ${ }^{3}$ Department of Biology, Rosenstiel Basic Medical Sciences Research Center, Brandeis University, Waltham, MA 02454, USA.

Correspondence and requests for materials should be addressed to T.A.K. (email: kunkel@niehs.nih.gov)
} 
R eplication of the undamaged eukaryotic nuclear genome is largely conducted by three members of the B family of DNA polymerases. DNA polymerase $\alpha$-primase synthesizes short RNA-DNA primers to initiate replication and DNA polymerases $\delta$ and $\varepsilon$ (Pols $\delta$ and $\varepsilon$ ) then perform the bulk of DNA chain elongation ${ }^{1-5}$. Because these polymerases only synthesize DNA in the $5^{\prime}$-to- $3^{\prime}$ direction, the leading strand of duplex DNA is normally replicated in a largely continuous fashion, while the lagging strand is synthesized discontinuously as Okazaki fragments. Considerable evidence now suggests that in the absence of stress, lagging-strand replication is primarily conducted by Pol $\delta$ and leading-strand replication is primarily conducted by Pol $\varepsilon$ (reviewed in ref. ${ }^{4}$ ). For example, a variant of Saccharomyces cerevisiae Pol $\varepsilon$ produces specific mismatches ${ }^{6}$ and incorporates an increased number of ribonucleoside triphosphates (rNTPs) ${ }^{7}$ during DNA synthesis in vitro, and these same behaviors are seen in vivo during leading-strand DNA replication in S. cerevisiae and Schizosaccharomyces pombe $e^{8-13}$. The analogous situation is true for variants of budding and fission yeast Pols $\alpha$ and $\delta$, whose strand-specific incorporation of both mismatches and ribonucleotides are consistent with lagging-strand replication ${ }^{10,11,14,15}$. This model is strongly supported by elegant studies of DNA replication catalyzed by yeast replication forks in vitro ${ }^{1-3}$. Moreover, it does not exclude that Pol $\delta$ is also involved in a smaller percentage of leadingstrand replication ${ }^{4}$. For example, elegant studies in $S$. pombe by Carr and colleagues suggest that Pol $\delta$ synthesizes about two kilobases of DNA on both DNA strands upon replication restart after pausing at the RTS1 locus ${ }^{16}$. They also reported a slight excess of ribonucleotides incorporated into the leading strand during replication by Pol $\delta^{13}$, and suggested that it may occasionally be recruited to initiate leading-strand replication. More recently, Diffley and colleagues have described a pulse-chase experiment in vitro of replication products made during replication with purified yeast proteins ${ }^{17}$, and they too have suggested that $\mathrm{Pol} \delta$ participates in initiating leading-strand replication.

On the other hand, studies performed in the 1990s revealed that SV40 origin-dependent DNA replication in vitro requires Pols $\alpha$ and $\delta{ }^{18-20}$, but not Pol $\varepsilon$. Moreover, S. cerevisiae pol2$16^{21-23}$ and $S$. pombe $c d c 20^{\Delta N-t e r m} 24$ mutant strains are viable despite having in-frame deletions of Pol $\varepsilon$ polymerase and exonuclease domains while leaving intact the $\mathrm{C}$-terminal domain that controls cellular responses to DNA damage. These facts are consistent with an alternative model that is supported by a recent study $^{25}$ proposing that Pol $\delta$ is the major replicase for both strands and that Pol $\varepsilon$ simply proofreads errors made by Pol $\delta$ as it replicates the leading DNA strand.

Here we provide evidence to distinguish between these two models and to inform our understanding of the initiation of nuclear DNA replication. We begin by examining the phenotypes of $S$. cerevisiae pol2-16 mutants in two strain backgrounds, and compare their phenotypes to those of pol2-4 mutants that lack exonuclease activity but retain polymerase activity $^{26}$. These comparisons support the idea that Pol $\varepsilon$ is the main DNA polymerase used to synthesize most of the undamaged leading strand during nuclear DNA replication in yeast. We then examine ribonucleotide incorporation into DNA in the pol2-16 mutant and conclude that in the absence of Pol $\varepsilon$ polymerization activity, Pol $\delta$ replicates both DNA strands, but does so poorly and with severe consequences on genome stability. Finally, we present ribonucleotide incorporation data with variants of Pols $\alpha, \delta$, and $\varepsilon$ that strongly support the hypothesis that Pol $\delta$ contributes to initiation of leading-strand replication in yeast by synthesizing DNA of both strands at replication origins.

\section{Results}

In vivo phenotypes of pol2-16 mutants. Seminal studies by Wittenberg and colleagues ${ }^{21}$ and by Campbell and colleagues ${ }^{22}$ demonstrated that $S$. cerevisiae strains containing an in-frame deletion of residues $176-1134$ of the Pol $\varepsilon$ catalytic subunit (pol216; Fig. 1a) are viable. Because this deletion removes the DNA polymerase and $3^{\prime}$-to-5' exonuclease activities of Pol $\varepsilon$ but leaves C-terminal residues intact, the survival of these pol2-16 strains demonstrates that yeast cells can replicate their nuclear genome in the absence of these two catalytic activities of Pol $\varepsilon$. In order to better understand the consequences of loss of Pol $\varepsilon$ catalytic activities on replication in yeast cells, we constructed the pol2-16 mutation and examined its properties. Because different genetic backgrounds can affect phenotype, and because this has been offered as one explanation for differences between our studies and that of Johnson et al. ${ }^{25}$, we constructed the pol2-16 mutant in two strain backgrounds. One is the yeast strain that we have previously used to investigate the role of Pol $\varepsilon$ in nuclear DNA replication, designated $\Delta 7$. The other is the commonly used strain background, W303. To distinguish between the two replication models, we compared pol2-16 phenotypes in both backgrounds to those of analogous wild-type yeast and to strains lacking Pol $\varepsilon$ 's 3 '-exonuclease activity (pol2-4) due to substitution of alanine for two negatively charged residues (D290 and E292) that are essential for proofreading of mismatches and ribonucleotides ${ }^{26,27}$. We anticipated that a comparison of the phenotypes of the pol2-16 and pol2-4 mutants would be informative regarding loss of the one or both of the catalytic activities of Pol $\varepsilon$ on survival, growth characteristics, and the role of Pol $\varepsilon$ in normal DNA replication.

We constructed heterozygous diploid yeast (pol2-16/POL2 and pol2-4/POL2) by replacing one of the POL2 alleles and explored the phenotypes of meiotic progeny. On the third day after tetrad dissection, wild-type and pol2-4 yeast formed haploid spore colonies that are visible by eye at both 23 and $30^{\circ} \mathrm{C}$ (Fig. 1b, c). At the same time, pol2-16 spore colonies are not visible to the naked eye, although micro-colonies are observable (Fig. 1d). After incubation for 12 days, some, but not all, of the pol2-16 mutants formed small spore colonies. In the $\Delta 7$ background, pol2-16 spore viability was $64 \%$ at $23{ }^{\circ} \mathrm{C}$ and $19 \%$ at $30{ }^{\circ} \mathrm{C}$; in the $\mathrm{W} 303$ background, the pol2-16 spore viability was $77 \%$ at $23^{\circ} \mathrm{C}$ and $44 \%$ at $30^{\circ} \mathrm{C}$. The differences in pol2-16 spore viability between $23^{\circ} \mathrm{C}$ and $30^{\circ} \mathrm{C}$ were statistically significant (Supplementary Fig. 1b). The viability of wild-type spores was not affected by temperature $-93 \%$ at both 23 and $30^{\circ} \mathrm{C}$ in the $\Delta 7$ background and $94 \%$ at $23^{\circ} \mathrm{C}$ and $93 \%$ at $30^{\circ} \mathrm{C}$ in the W303 background (Supplementary Fig. 1c). Freshly isolated pol2-16 spore colonies have doubling times that are strongly increased as compared to the pol2-4 mutant and wild-type yeast (Fig. 1e).

Western blot analysis of Pol2p levels revealed that the truncated Pol2 $\mathrm{p}$ in the pol2-16 yeast is unstable, which manifest as multiple bands of degradation products (Fig. If and Supplementary Figs. 5 and 6). The level of non-degraded Pol2p in pol2-16 yeast is about $70 \%$ lower than wild type (Fig. 1g).

Consistent with earlier studies ${ }^{21-23}$, our pol2-16 mutants have larger cell sizes than wild type (Fig. 2a), and they progress more slowly through the cell cycle (Fig. 2b). Moreover, the DNA content of pol2-16 mutant cells is higher than that of wild-type or pol2-4 mutant cells (Supplementary Fig. 1d), and this DNA is aberrantly distributed (Fig. 2a and Supplementary Fig. 1e). When pol2-16 spore colonies were resuspended and plated onto complete medium, colonies formed that ranged from barely macroscopic to close to wild-type in size (Fig. 2c). This indicates rapid accumulation of suppressors that improve pol2-16 fitness (parental suppressors are precluded; Supplementary Tables 1 and 2). To minimize the effects of such suppressors, subsequent 
a

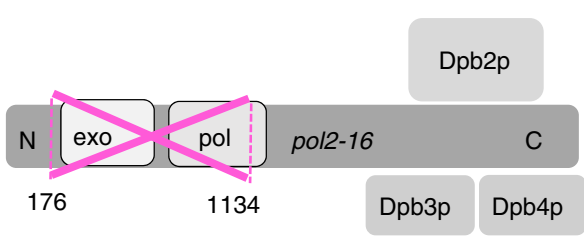

b

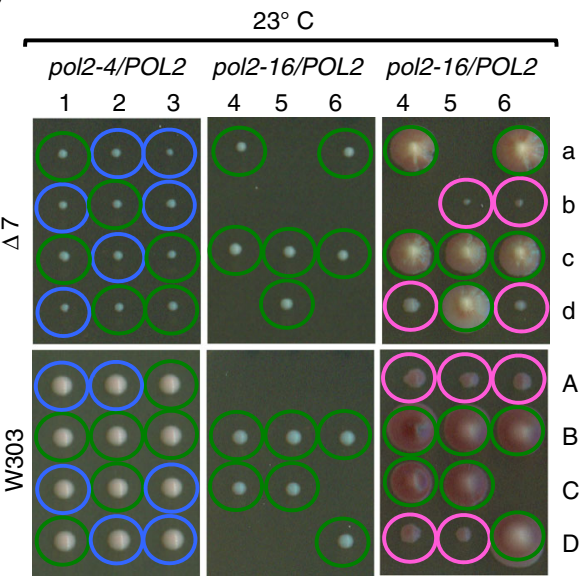

3 days

12 days

C

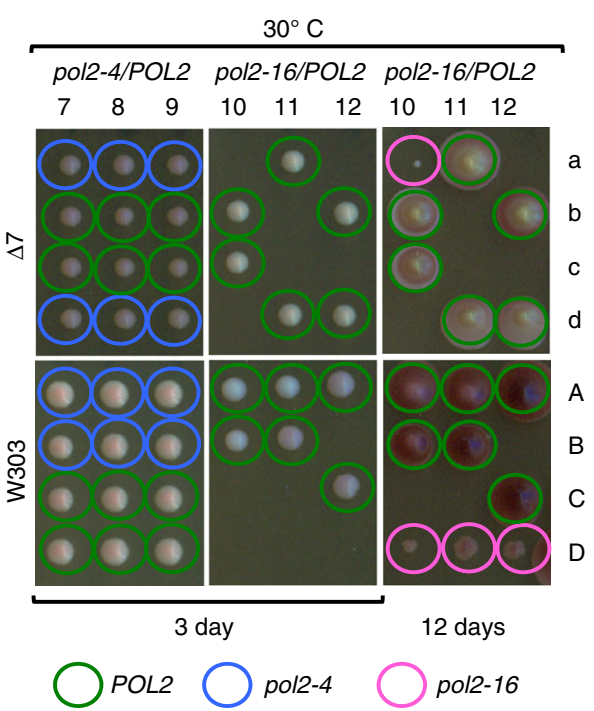

d

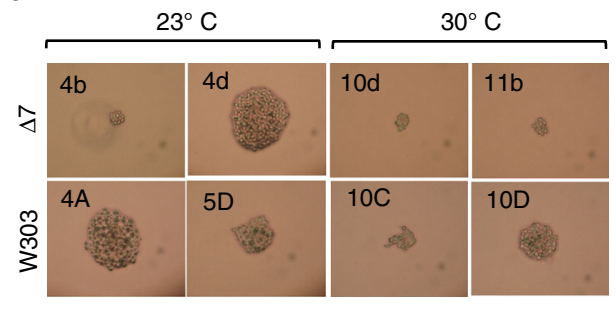

e

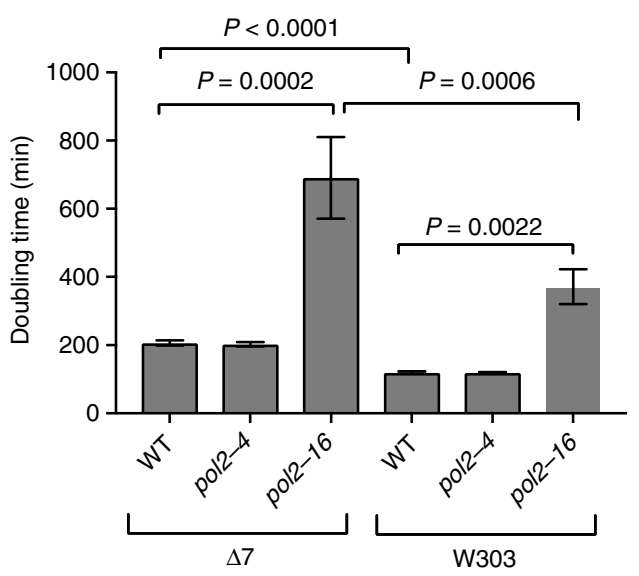

f

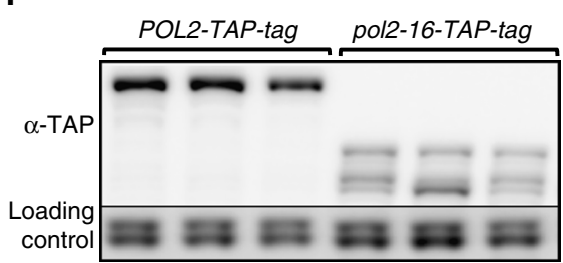

g

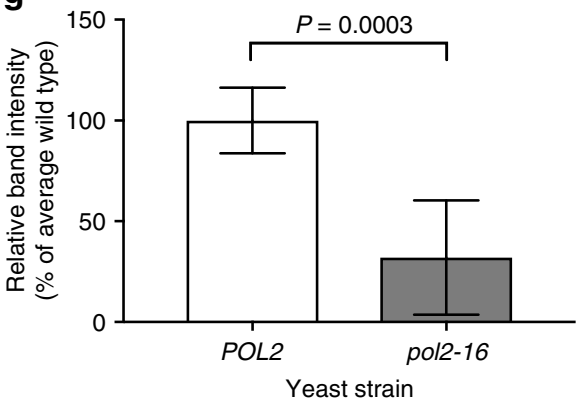

Fig. 1 Pol $\varepsilon$ catalytic domains are critical for yeast growth. a Schematic representation of DNA Polymerase $\varepsilon$ (Pol $\varepsilon$ ). The S. cerevisiae holoenzyme consists of the catalytic subunit (Pol2p) and three auxiliary subunits: Dpb2p, Dpb3p, and Dpb4p ${ }^{52-55}$. Cryo-electron microscopy has shown that Pol2p has two lobes tethered by a flexible linker ${ }^{43}$. Active polymerase and exonuclease domains are in the $\mathrm{N}$-terminal lobe. The pol2-16 mutant has an in-frame deletion of the fragment of catalytically active lobe (amino acids 176-1134). b, c Tetrad analysis of pol2-16/POL2 and pol2-4/POL2 heterozygous diploids in two yeast backgrounds, $\Delta 7$, and $\mathrm{W} 303$, at $23^{\circ} \mathrm{C} \mathbf{b}$ and $30^{\circ} \mathrm{C}$ c. 1-12 are dissected tetrads, $A-D$, and a-d are haploid spore colonies. Images were taken after 3 and 12 days. Genotypes were confirmed via PCR (pol2-16, red circles) or sequencing (pol2-4, blue). Wild-type colonies are circled in green. The lack of both Pol $\varepsilon$ catalytic domains (pol2-16) causes severe growth defects. Exonuclease inactivation alone (pol2-4) does not. $\mathbf{d}$ Microscopic images of pol2-16 colonies taken 3 days after tetrad dissections. e Doubling times of po/2-16 and po/2-4 mutants compared to wild-type yeast. Doubling times were estimated from optical density at $600 \mathrm{~nm}$ of cultures grown at $23^{\circ} \mathrm{C}$. Error bars represent standard deviations $(n=4-6$ yeast cultures, two or three from two independent isolates). Unpaired two-tailed $t$ tests with Welch's correction yielded $p$ values $(P)$. The doubling time of the pol2-16 mutant is about threefold longer than of the wild-type and pol2-4 yeast in both $\Delta 7$ and W303 backgrounds. The difference in the doubling times between the wild-type $\Delta 7$ and W303 backgrounds may be due to one or more of over 10,000 SNPs detected by the whole-genome sequencing. $\mathbf{f}$ Western blot detection of Pol2p level in whole-cell extracts. Presented are bands for three independent isolates of strains bearing POL2 or pol2-16 in fusion with TAP-tag. Immunoblotting was performed using an antibody to TAP-tag or PSTAIR (loading control). $\mathbf{g}$ Relative band intensity. Error bars represent standard deviations $(n=6-7$ independent yeast isolates). Unpaired two-tailed $t$ tests with Welch's correction yielded $p$ values $(P)$ 
a
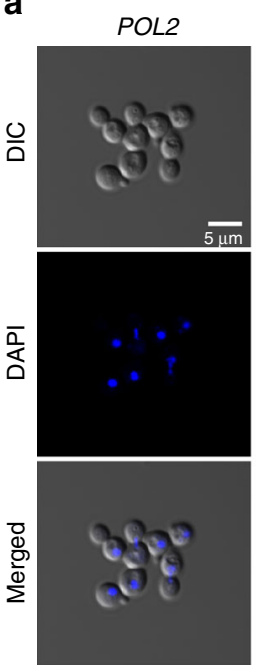

西
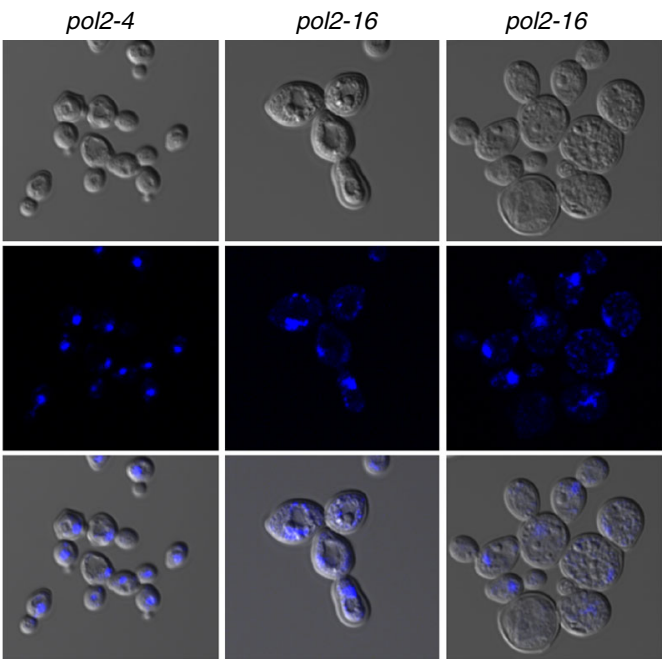

b

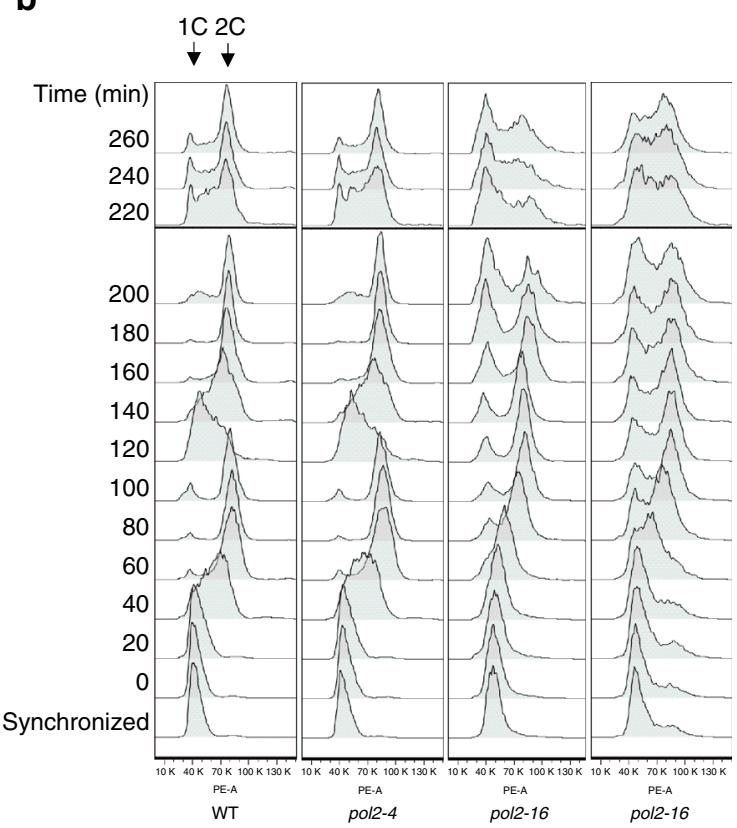

\section{C}
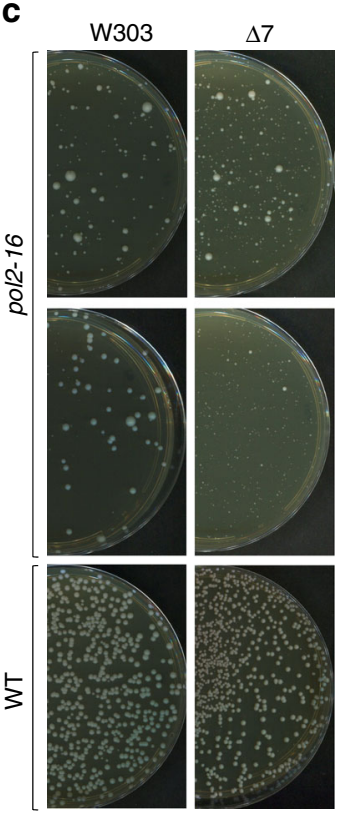

Fig. 2 Phenotypes of wild-type, pol2-4, and pol2-16 mutants. a Confocal microscope images of DAPI stained, exponentially growing yeast in the synthetic complete media, fixed with 70\% ethanol. The pol2-16 cells are much bigger than wild-type and pol2-4 cells and have aberrantly distributed DNA. b Flowcytometric analysis of cell cycle progression. Yeast cells were $\alpha$-factor arrested in $\mathrm{G} 1$ phase, then released into complete media, sampled every 20 min, fixed with 70\% ethanol, stained with propidium iodide, and then analyzed with flow cytometry (Methods). $1 \mathrm{C}$ and $2 \mathrm{C}$ indicate the DNA contents. c Colony size heterogeneity in the pol2-16 outgrowths. Resuspended pol2-16 and POL2 spore colonies, were plated on solid complete media and incubated at $23^{\circ} \mathrm{C}$ for 6 and 4 days, respectively

experiments (with one exception, see below) were performed with pol2-16 spore colonies freshly isolated from progeny of heterozygous pol2-16/POL2 diploids.

Replication enzymology in the W303 background. We examined the genome-wide density of ribonucleotides incorporated by variants of the three major replicases, Pol a (pol1-L868M), Pol $\delta$ (pol3-L612M), and Pol $\varepsilon$ (pol2-M644G) in the W303 strain background. Relative to their wild-type parents, these polymerases all have an elevated ability to incorporate ribonucleotides into $\mathrm{DNA}^{7,27}$. This property can be visualized when ribonucleotide excision repair (RER), the primary mechanism of ribonucleotide removal, is absent (i.e., in the rnh2014 background; reviewed in ref. ${ }^{10}$ ). This in vivo analysis uses HydEn-seq ${ }^{11}$, a procedure that maps the locations of $5^{\prime}$ DNA ends via paired-end sequencing of genomic DNA after alkaline hydrolysis. In $r n h 201 \Delta$ strains, these positions largely indicate the locations of ribonucleotides incorporated by each DNA polymerase during replication, in a strand-specific manner. We therefore performed a meta-analysis of HydEn-seq data to examine ribonucleotide incorporation around 214 well-characterized replication origins in the W303 strain background ${ }^{28}$. The DNA ends in the pol1-L686M rnh2014 and pol3-612M rnh2014 strains were preferentially found in the newly synthesized lagging strand (Fig. 3a, c and Supplementary Data 1 and 2). In contrast, in pol2-M644G rnh2014, DNA ends were primarily present in the nascent-leading strand (Fig. 3b and Supplementary Data 1 and 2). 
a

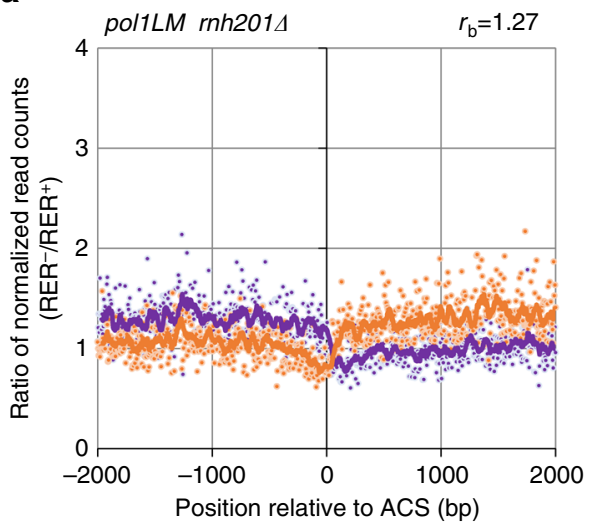

b

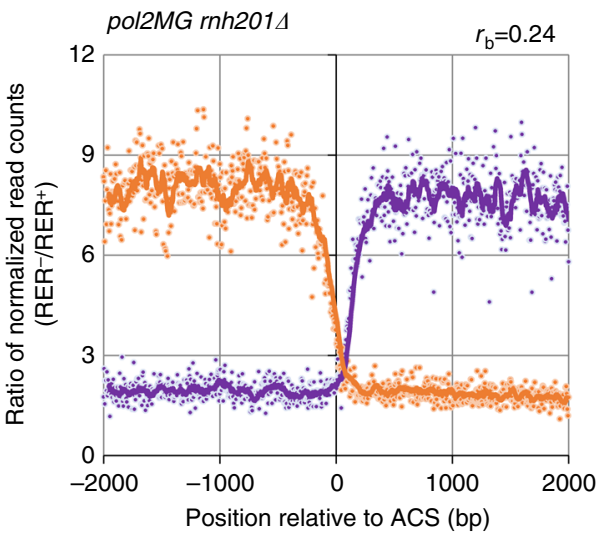

C

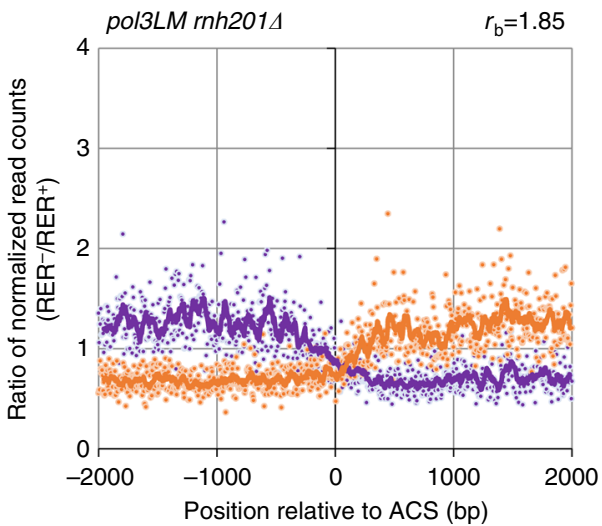

$\mathbf{d}^{\prime}$

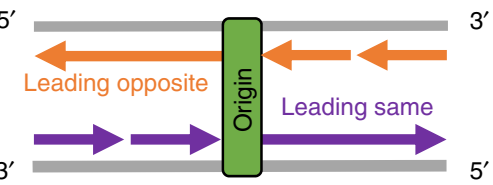

Same

Opposite

e

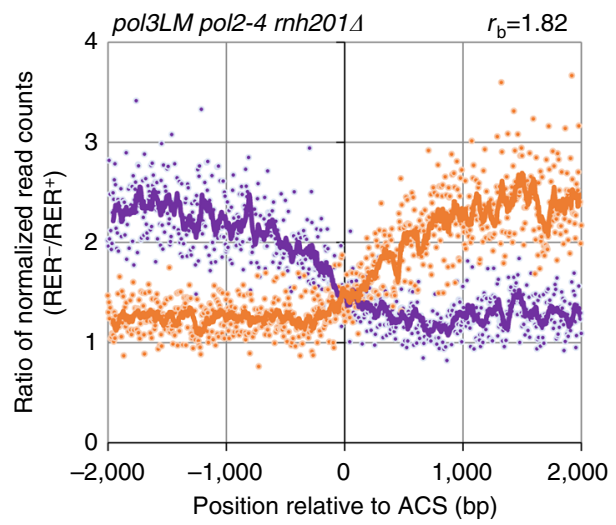

$\mathbf{f}$

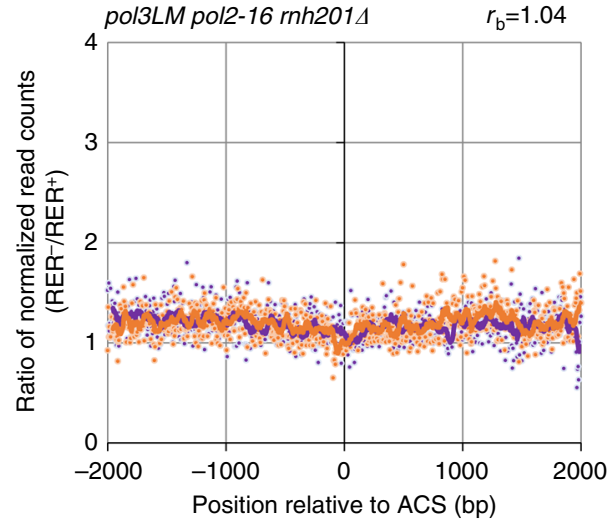

Fig. 3 Ribonucleotides as biomarkers of replicase activity. Meta-analysis of ribonucleotides abundance in the vicinity of 214 replication origins analyzed in previous study ${ }^{28}$, in bins of $5 \mathrm{bp}$, with 10-bin moving average trend lines. Read counts were normalized as described in Supplementary Methods. Shown are ratios of RER-deficient (RER ${ }^{-}$) and RER-proficient (RER ${ }^{+}$) strains bearing a pol1L868M, b pol2M644G, c pol3L612M, e pol3LM pol2-4, and $\mathbf{f}$ pol3LM pol2-16 mutations. Values on the right above each chart are average lagging-over-leading strand biases $\left(r_{b}\right)$ (see calculations in Supplementary Methods). For each panel, a representative of two independent measurements is presented. $\mathbf{d}$ Schematic representation of DNA replication in the vicinity of replication origin, depicting the leading and lagging strands orientations

Pol $\delta$ synthesizes both strands in the pol2-16 mutant. In pol2-16 mutants lacking Pol $\varepsilon$ polymerase activity, Pol $\delta$ is a logical candidate for replicating leading-strand DNA. To test this possibility, we next used HydEn-seq to compare strand-specific genomic ribonucleotide abundance in pol3LM rnh201A strains containing either wild-type Pol $\varepsilon$, the pol2-4 mutation, or the pol2-16 mutation. As mentioned above, in the W303 doublemutant pol3L612M rnh201S strain, significantly more ribonucleotides are observed in the lagging strand (Fig. 3c), with a lagging-over-leading strand bias of 1.85 (calculations in
Supplementary Methods). A similar result was observed for the pol3L612M rnh2014 pol2-4 strain (Fig. 3e). These results are consistent with Pol $\delta$ being the major lagging-strand replicase and demonstrate that the leading-strand biases observed in the pol2M644G rnh201د mutant are due to Pol $\varepsilon$ polymerase activity rather than to defective Pol $\varepsilon$ proofreading of Pol $\delta$ errors on the leading strand. However, in the triple mutant pol3L612M rnh2014 pol2-16 strain, the strand bias is greatly reduced (1.04; Fig. 3f). These results suggest that Pol $\delta$ replicates both nascent strands in the absence of Pol $\varepsilon$ catalytic activity, albeit with 
reduced efficiently that manifests as slow growth phenotypes for pol2-16 mutants (Figs. 1 and 2).

Pol32 and Pif1 are required in the pol2-16 mutant. When Pol $\varepsilon$ is inactivated, kilobases of break-induced replication (BIR) synthesis are accomplished in a Pol $\delta$-dependent manner ${ }^{29}$. While the Pol32 subunit of Pol $\delta$ and the $5^{\prime}-3^{\prime}$ Pifl helicase are not required for normal DNA replication, they are required for BIR, where recombination establishes a non-canonical replication fork $^{29-31}$. To see whether Pol32 and Pif1 are required for extensive leading-strand DNA replication by Pol $\delta$ in the pol2-16 mutant, we performed the series of crosses outlined in the Supplementary Fig. 3d. In this series of crosses, during propagation of the initial ASY102 pol2-16 strain, as well as of haploid strains obtained from tetrad dissections, unknown suppressor(s) of the pol2-16 slow growth phenotype could have been acquired. The distribution of spore phenotypes from a subsequent cross (Supplementary Figs. 3a and 4) is in agreement with a single suppressor that segregates independently of pol2-16 in this diploid (Supplementary Tables 3 and 4). Two different fast-growing pol216 isolates from progeny of heterozygous pol2-16/POL2 diploids were crossed with pol32 $\Delta$ or with pif1 $\Delta$ isolates from the same series of crosses (Supplementary Fig. 3b, c). No pol2-16 pol32 meiotic segregants arising from a cross were viable. In contrast, half of the pol2-16 segregants, presumably those with the suppressor, grew well (Supplementary Fig. 3). The pol2-16 pifis double-mutant segregants did grow, but grew poorly.

Pol $\delta$ participates in leading-strand replication at origins. We observed that ribonucleotide density at origins is elevated in both nascent strands in the strains bearing Pol $\delta$ variants and minimal in the pol2-M644G rnh2014 strains, in both the W303 (Supplementary Fig. 2 and Supplementary Data 2) and $\Delta 7$ strain backgrounds ${ }^{11}$. Based on these observations, we decided to further explore the replication enzymology of the leading strand in vivo to test the possible involvement of Pol $\delta$ in leading-strand initiation. After subtracting the HydEn-seq end densities of the RER-proficient strains from their RER-deficient analogs (Supplementary Methods, Supplementary Fig. 2, and Supplementary Data 2), we solved a system of simultaneous equations to account for ribonucleotide incorporation by each variant polymerase during replication across origins (Supplementary Methods). This meta-analysis used 214 well-behaved replication origins, centered on the $5^{\prime}$-end of the autonomously replicating sequence (ARS) consensus sequence (ACS). The results (Fig. 4) indicate that the fraction of DNA synthesis conducted by each variant polymerase differs immediately proximal to the point of initiation. As an example, for the most promiscuous DNA polymerase for ribonucleotide incorporation, Pol $\alpha^{7}$, the total amount of DNA synthesis at the origin (Fig. $4 \mathrm{a}$, in red) is low because the DNA primers it synthesizes are only about 10-20 nucleotides long. When the results with Pol $\alpha$ are used to estimate where Pol $\alpha$ begins to synthesize DNA at an average replication origin, the results reveal a peak of DNA synthesis centered just upstream of the ACS (Fig. 4b). On the other hand, Pol $\varepsilon$ synthesizes much more DNA during replication and this synthesis is maximal by about 300 nucleotides downstream of the ACS (blue line in Fig. 4a). This is consistent with its role as the major leadingstrand replicase. However, the beginning of this synthesis peaks about 180 nucleotides downstream of the ACS (Fig. 4a, b), approximately one Okazaki fragment length after the peak for initiating short primers synthesized by Pol $\alpha$.

At locations $>400$ nucleotides upstream of the ACS (left of the ACS in Fig. 4a, b), the ribonucleotide incorporation data indicate that total synthesis by Pol $\delta$ (green in Fig. 4a) is maximal and almost as high as the maximum for Pol $\varepsilon$ seen on the leading strand. This pattern is anticipated by the model wherein Pol $\delta$ primarily replicates the lagging strand while incorporating ribonucleotides at the lowest rate among the three replicases ${ }^{7}$. More interestingly, after subtracting canonical lagging-strand synthesis (Supplementary Methods, Eq. 20, and Supplementary Data 3), a peak of Pol $\delta$ synthesis remains, with the beginning of this tract peaking about 10 nucleotides downstream of the Pol a initiation peak, well before the beginning of synthesis by Pol $\varepsilon$. Moreover, the standard deviation for the Pol $\delta$ tract length is much smaller than the track length $(0-12 \ll 140-180)$, indicating that Pol $\alpha$-to-Pol $\varepsilon$ transfers ( $\mathrm{Pol} \delta$ tract $=0$ ) are exceedingly rare. These results suggest that Pol $\delta$ synthesizes both DNA strands over a short distance at most replication origins, after which synthesis of the leading strand is primarily conducted by Pol $\varepsilon$.

\section{Discussion}

Studies by Kesti et al. ${ }^{21}$ and by Dua et al. ${ }^{22}$ showed that pol2-16 is able to complement the lack of growth at increased temperature of yeast strains bearing temperature-sensitive alleles of pol2. Based on that analysis, they concluded that the Pol $\varepsilon$ catalytic domains are dispensable for DNA replication and cell viability. However, the time between haploid strain construction and commencement of complementation experiments risks acquisition of suppressor mutations that could mask the full effects of pol2-16. We observed an apparent rapid acquisition of suppressors in spore colonies, and thus decided to minimize the number of generations during which yeast cells experience selective pressure against the effects of pol2-16. We constructed pol2-16/POL2 heterozygous diploids from which we isolated haploid pol2-16 meiotic progeny to measure phenotypes while minimizing accumulation of suppressors. Kesti et al. ${ }^{21}$ also created pol2-16/POL2 heterozygous diploids and observed that pol216 spores "often germinated but grew into colonies only infrequently ( $10 \%$ of expected segregants)." Kesti et al. ${ }^{21}$ suggested that these phenotypes reflect the importance of the $\mathrm{N}$-terminal catalytic activities of Pol $\varepsilon$ during the first few cell cycles. Given the rapid suppressor accumulation that we observe here, we do not disagree with this interpretation. However, we further suggest that these phenotypes reflect the importance of Pol $\varepsilon$ for replicating leading-strand DNA in all cell divisions, with the chance of observing the phenotypes of the pol2-16 mutation being highest before suppressors come to dominate the population. In the future, it will be interesting to understand the nature of the suppression that is occurring in pol2-16 cells and whether it is due to acquisition of mutations, epigenetic changes, or non-encoded metabolic adaptations that improve fitness.

The decreased level of Pol2p in the pol2-16 freshly isolated spore colonies suggests that the truncated Pol2p in pol2-16 is unstable. This suggests that the N-terminal portion of Pol2p (bearing the catalytic domains) is critical to stabilize the Cterminal part of Pol2p that is essential for yeast viability. It may further suggest that the sickness/near-inviability of pol2-16 yeast could be due to replication initiation defects. The C-terminal part of Pol2p interacts with Dpb2p, which bridges the interaction of Pol $\varepsilon$ with the GINS complex ${ }^{32-34}$. Pol $\varepsilon$ and GINS are components of the CMGE helicase polymerase whose formation is essential for initiation of chromosomal DNA replication ${ }^{32,33,35}$.

Overall, our studies of the pol2-16 variant of Pol $\varepsilon$ performed in two yeast strain backgrounds imply that the complete loss of Pol $\varepsilon$ polymerase and exonuclease domains yields tiny spore colonies, rapid suppressor accumulation, greatly increased doubling time, increased cell size and aberrant DNA distribution. These phenotypes are not observed in the strain bearing the pol2-4 mutation that lacks only Pol $\varepsilon$ 's proofreading activity. These data are 
a

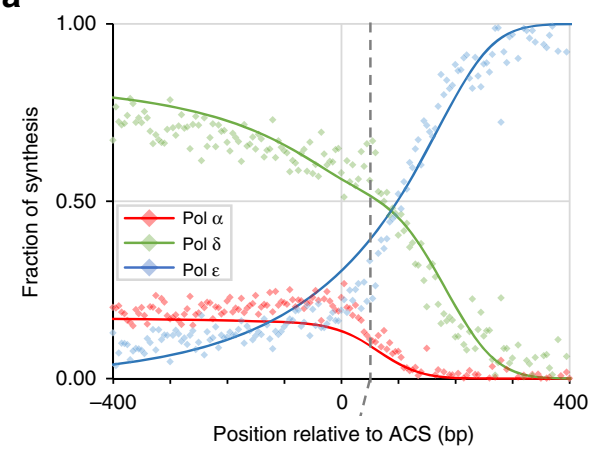

C

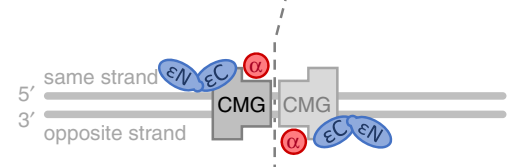

e

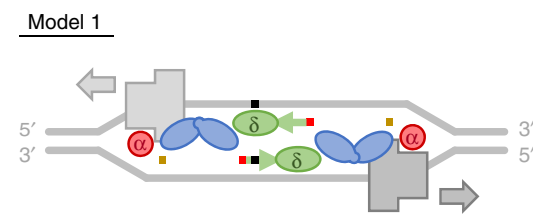

f

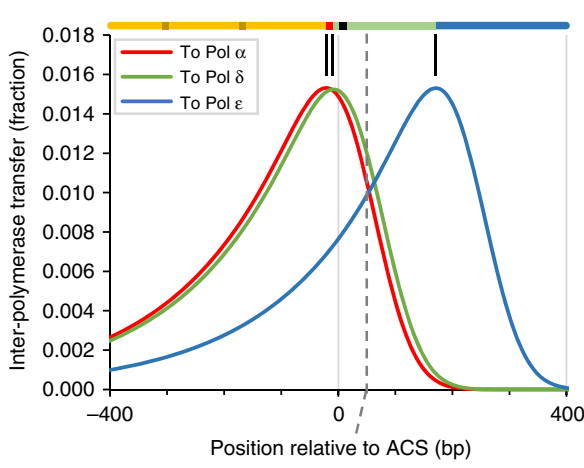

d

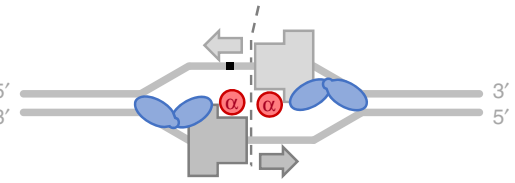

Model 2
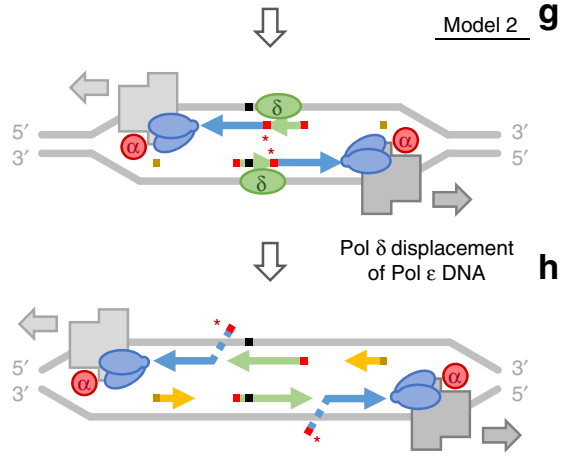

Nick translation and/or flap excision

i

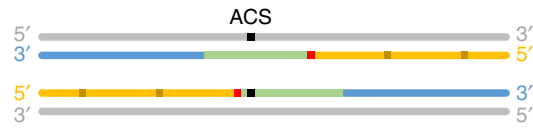

Fig. $4 \mathrm{HydEn-seq-derived} \mathrm{evidence} \mathrm{for} \mathrm{DNA} \mathrm{polymerase} \delta$ participation in leading-strand synthesis at yeast origins. Red, green, and blue denote polymerases $\alpha, \delta$, and $\varepsilon$, respectively, or DNA tracts synthesized by same. Canonical Okazaki fragments (yellow), synthesized by Pols $\alpha$ (dark), and $\delta$ (light) are approximately positioned exemplars. S. cerevisiae origins (identified by Smith et al. ${ }^{28}$ ) are oriented such that ARS consensus sequences (ACS) are $5^{\prime}-3^{\prime}$ beginning at position 0. a Diamonds represent the fraction of DNA strand synthesized by DNA Pols $\alpha, \delta$, and $\varepsilon$ ( 5 bp bins; calculated from rescaled and background subtracted HydEn-seq end densities in pol1-L868M rnh2014, pol3-L612M rnh2014, and pol2-M644G rnh2014 strains; see Supplementary Methods). Data for both strands were averaged (opposite strand reflected around $+45 \mathrm{bp}$, the axis of strand symmetry; gray dashed line). Solid curves are regression models. $\mathbf{b}$ The fraction of inter-polymerase transfer events outside of canonical Okazaki fragment synthesis (extracted from regression models). The mode of each curve (vertical black line) suggests the most frequent synthesis tract (colored bars above). c-i Schematics of two non-exclusive models of polymerase action at yeast replication origins. DNA strands (colored bars) have the same horizontal scale as in $\mathbf{a}$ and $\mathbf{b}$; polymerases (ellipses) and CMG helicases (gray polygons) are exaggerated; other components are omitted. $\varepsilon \mathrm{N}$ and $\varepsilon \mathrm{C}$ indicate $\mathrm{N}$-terminal catalytic and C-terminal CMG-binding Pol $\varepsilon$ domains. c Head-to-head dsDNA-binding CMG helicases. d Helicases transition to ssDNA-binding and translocate past one another, $\mathrm{N}$-termini facing directions of travel (gray arrows). e, $\mathbf{f}$ Model 1. e Pol $\alpha$ associated with each replisome primes the leading strand that will be synthesized by the other ( $0.1 \%$ probability per bp translocated, from regression). Pol $\delta$ extends each nascent-leading strand. $\mathbf{f}$ Pols $\delta$ collide with respective replisomes, releasing $3^{\prime}$-termini to Pols $\varepsilon$. They assuming synthesis conformation to extend the leading strand. $\mathbf{g}$, h Model 2. $\mathbf{g}$ Unidentified Pols $\alpha$ prime leading-strand replication ( ${ }^{\star} ;$ extended by Pols $\varepsilon$ ). The Pol $\alpha$ associated with each replisome primes the first Okazaki fragment (extended by Pol $\delta$; destined to be longer than average). $\mathbf{h}$ Pol $\delta$ displaces the $5^{\prime}$ primer terminus of the nascent-leading strand, allowing nick translation or flap excision. i Synthesis patterns from both pathways indicate apparent Pol $\delta$ synthesis of both nascent strands at the origin

consistent with the hypothesis that Pol ع's polymerase activity, but not its proofreading activity, is crucial for efficient replication of undamaged chromosomal DNA that results in normal cell growth. These observations in vivo are in agreement with the recent in vitro data showing that full-length Pol $\varepsilon$ is required for the maximal rate of leading-strand DNA replication ${ }^{17}$.

The idea that Pol $\varepsilon$ is the major leading-strand replicase has been questioned by Johnson et al. ${ }^{25}$, who have suggested that differences in $S$. cerevisiae strain backgrounds could be misleading and that Pol $\delta$ is instead the major replicase for both the leading and lagging strands. We do not consider this explanation to be likely given results in $S$. cerevisiae and $S$. pombe as well as mutation asymmetry around human origins in POLE-exo- ${ }^{-}$cancers, all of which support the idea that Pol $\varepsilon$ is the major leadingstrand replicase ${ }^{6,9,11,13-15,36-38}$. Nevertheless, because our previous studies were performed in the $\Delta 7$ strain background, we now include the W303 background when testing the two models. Our new ribonucleotide-incorporation data from the W303 background are remarkably similar to results in the $\Delta 7$ strain background $^{11}$, and are consistent with Pol $\alpha$ and Pol $\delta$ as primary 
lagging-strand replicases and Pol $\varepsilon$ as the primary leading-strand replicase.

Yeast are viable without Pol $\varepsilon$ catalytic domains (pol2-16) but inviable with point mutations that abrogate Pol $\varepsilon$ polymerase activity (pol2-D875A D877A) ${ }^{22}$. This suggests that at least one other polymerase can replicate the leading strand unless physically excluded from the primer terminus by inactive Pol $\varepsilon$. In the presence of PCNA, Pol $\delta$ can processively synthesize at least five kilobases of RPA-coated single-stranded $\mathrm{DNA}^{39}$, which makes it the most probable polymerase to substitute for Pol $\varepsilon$ in synthesis of the leading strand. Indeed, when replication fork progression is impeded at the RTS1 locus in $S$. pombe, replication restarts through initial synthesis of both DNA strands by Pol $\delta^{16}$. The strand-specific ribonucleotide density in the pol3LM rnh201A pol2-16 strain revealed no strand bias when the Pol $\varepsilon$ catalytic domains are missing (Fig. 3), unlike the high bias in the pol3LM $r n h 201 \Delta$ strain, thereby suggesting that Pol $\delta$ replicates both the leading and lagging strands across entire genome when $\operatorname{Pol} \varepsilon$ is not present. Bulk leading-strand replication by Pol $\delta$ is very inefficient, which manifests as elongated S-phase and doubling time of yeast bearing the pol2-16 mutation, likely due to a reduced rate of DNA unwinding by CMG that requires catalytic domain of Pol $\varepsilon$ for the maxim unwinding rate ${ }^{17}$. Additionally, analysis of the synthetic genetic interactions of pol2-16 with pif1 $\Delta$ and pol32 $\Delta$ suggest that for Pol $\delta$ to carry out extensive leading-strand synthesis, cells become reliant on normally non-essential replication factors such as Pif1 or Pol32. It is possible that the requirement for POL32 and PIF1 when Pol $\varepsilon$ catalytic activity is absent is similar to their requirement in extensive DNA synthesis during BIR, where leading and lagging-strand DNA synthesis are not coupled ${ }^{31}$ and where the initial DNA synthesis appears to be dependent on Pol $\delta$, with Pol $\varepsilon$ only being required at a later stage $^{29,40}$. Further work will be needed to determine if the poor growth of the pol2-16 pif1 $\Delta$ double mutant is partially due to loss of mitochondrial DNA due to PIF1 deletion ${ }^{41}$.

The idea that Pol $\varepsilon$ is the major polymerase for leading-strand replication of undamaged DNA does not exclude Pol $a$ or Pol $\delta$ participation in some fraction of leading-strand replication. Current mutagenesis and ribonucleotide incorporation data and eSPAN analyses ${ }^{42}$ set the upper bound for such participation in yeast at between 2 and $23 \%$, with the preponderance of evidence supporting the lower end of that range 4 . Using ribonucleotide bin sizes of 300 bases, Carr and colleagues reported that $S$. pombe Pol $\delta$ may occasionally initiate leading-strand replication ${ }^{13}$. More recently, a pulse-chase experiment of DNA synthesis by a minimal yeast replisome reconstituted using purified proteins and a single replication origin ${ }^{17}$ led to the same interpretation. Our high-resolution ribonucleitide incorporation data in vivo (5-bp bins; Fig. 4b) are consistent with these two studies, and strongly imply that Pol $\delta$ synthesizes both nascent strands at most replication origins over a distance of about 160 nucleotides. Elegant biochemical data that include cryo-electron microscopy structures of the minimal replication fork ${ }^{17,43,44}$ imply that nuclear DNA replication in budding yeast is initiated when two CMG helicases bind to DNA at origins (Fig. 4c). A Pol a-primase complex (red) binds via Ctf4 (not shown) to the leading face of the CMG, relative to the direction of fork motion. On the trailing face of each CMG is a four-subunit Pol $\varepsilon$ holoenzyme complex (blue). The catalytic Pol $\varepsilon \mathrm{N}$-terminal regions $(\varepsilon \mathrm{N})$ are not yet bound to DNA in an enzymatically productive manner. The Pol $\varepsilon$ C-terminal region is bound to CMG via GINS (not shown). After initial binding to duplex DNA (Fig. 4c), the two CMG complexes transition to bind single-stranded DNA (Fig. 4d) while moving past each other in opposite directions in an ATP-dependent manner. Two ssDNA templates are then available for Pol $\alpha$ to initiate replication on opposite DNA strands. Based on these facts, we suggest two possible models for Pol $\delta$ involvement into the initiation of leading-strand replication.

In the first model, Replication Factor C (RFC) first loads Proliferating Cell Nuclear Antigen (PCNA) (not shown) onto the primers made by Pol $\alpha$ (centered red bars in Fig. 4e). Pol $\delta$ (green) then binds and rapidly synthesizes nascent-leading strand DNA in a PCNA-dependent manner (Fig. 4e). This synthesis proceeds for about 160 nucleotides (Fig. 4b), until Pol $\delta$ approaches Pol $\varepsilon$ associated with the other CMG, which has not yet encountered a primer terminus from which to begin synthesis. Pol $\delta$ then undergoes "collision release"17,45,46. This allows the N-terminal catalytic domain of Pol $\varepsilon$ to engage the primed DNA (Fig. 4f) and, using PCNA as a co-factor ${ }^{17,47}$, to begin rapid and processive synthesis of leading-strand DNA. Given that the rate of replication is rapid at about 2000 nucleotides per min (ref. ${ }^{17}$ and references therein), these processes could all occur within a few seconds. This is the mechanism of initiation suggested by Diffley and colleagues earlier this year (ref. ${ }^{17}$ and reviewed in ref. ${ }^{3}$ ) based on in vitro studies of the minimal replication complex.

In the second model, Pol $\varepsilon$ could extend from the initial primer laid down by Pol a (Fig. 4g). This idea is central to many previous studies. The initial primer synthesized by Pol a (labeled ${ }^{\star}$ in Fig. 4g, h) would have to be completely excised via extensive nick translation synthesis, presumably performed by the Pol $\delta$ that extended from the next Pol a priming event (the first, noncanonical Okazaki fragment; Fig. 4g, h). Note that there is as yet no direct evidence for such extensive nick translation by Pol $\delta$, and given the relative positions of Pol $\alpha$ and Pol $\varepsilon$ in replisome structures, it is difficult to see how Pol a associated with one CMG could directly prime synthesis by Pol $\varepsilon$ associated with the other CMG. For these reasons, we favor the first model for Pol $\delta$ participation in initiation. That said, both models are consistent with the HydEn-seq data (Fig. 4i) and the two models are not mutually exclusive. Both mechanisms could operate depending on yet to be determined circumstances.

The data presented here are consistent with two main ideas. First, in contrasting pol2-4 and pol2-16 mutants (Figs. 1 and 2) in two strain backgrounds, it is clear that the compromised phenotypes and ribonucleotide strand biases of the pol2-16 mutant must primarily result from loss of Pol $\varepsilon$ 's polymerase activity during leading-strand synthesis, rather than from loss of its proofreading exonuclease. Therefore, given the leading-strand ribonucleotide incorporation bias (Fig. 3b), as well as a preponderance of previous studies, Pol $\varepsilon$ is the major DNA polymerase used to replicate the leading strand of the eukaryotic nuclear genome. Second, HydEn-seq end densities indicate that Pol $\delta$ contributes to the synthesis of both nascent strands immediately at origins, likely by initiating leading-strand replication.

\section{Methods}

Yeast strains construction. S. cerevisiae strains used in this study are listed in Supplementary Data 1 . All yeast strains (except strains used to analyze genetic interactions of pol2-16 with pif1 $\Delta$ and pol32 $\Delta$, see below) were isogenic derivatives of either $\Delta|(-2)|-7 B-Y U N I 300$, representing the $\Delta 7$ background, or AC402 and AC403, representing the W303 background. Diploids of $\Delta 7$ background were generated from haploid strain using YEpHO as described earlier ${ }^{14}$. Wild-type diploids of W303 background were generated by crossing AC402 and AC403 haploids.

The pol2-16 mutation was introduced to diploid S. cerevisiae via two-step gene replacement, using YIPlac211-pol2-16 plasmid, linearized with BlpI to target integration to the POL2 locus. $\mathrm{Ura}^{+}$recombinants were selected on SC-URA and toothpicked twice onto 5-FOA plates to select pol2-16/POL2 that have excised the plasmid. The presence of the pol2-16/POL2 alleles was confirmed by PCR (primers: POL2_-391_f, POL2_1102_r, and POL2_695_r; PCR product size: POL2 - 1509 bp, pol2-16 - 1102 bp). To exclude the presence of single nucleotides changes in the pol2-16 locus, the pol2-16 locus in the heterozygous diploids was amplified as two fragments ( $\mathrm{N}$ and $\mathrm{C})$. Primers used to amplify fragment $\mathrm{N}$ : POL2_-391_f, POL2_695_r, and fragment C: POL2_3423_f and POL2_8156_r. Sequencing 
results were analyzed using CLCGenomics Workbench 8.5.1. Primers sequences are listed in Supplementary Data 1.

The yeast bearing pol2-4, pollL868M, pol2M644G, or pol3L612M polymerases variants were constructed via integration-excision method using plasmids: YIpJB1 ${ }^{26}$, pYIAL30-pol1L868M ${ }^{8}$, p173-pol2M644G ${ }^{6}$, and p170-pol3L612M ${ }^{8}$, respectively.

Strains with deletion of RNH201 ( $r n h 201 \Delta$ ) were constructed using one-step gene disruption. PCR product containing hygromycin B-resistance cassette $(H P H)$ and region about $300 \mathrm{nt}$ upstream and downstream of RNH201 was amplified from genomic DNA of SNM106 using primers: RNH35-5'_flank_f and RNH-3'_flank_r. The presence of $r n h 201 \Delta:: H P H$ in transformants that were $\mathrm{Hygr}^{\mathrm{R}}$ was confirmed by PCR using primers: intHPH-3' ${ }^{\prime}$ for and RNH35-3' ${ }^{\prime}$ down1.

Strains with TAP-tag were constructed using POL2-TAP-tag-HIS3MX6 cassette amplified using primers: POL2_6425_f and POL2_6902_r from genomic DNA of YSC1178-202233129 (Dharmacon). The presence of TAP-tag in transformants that were $\mathrm{HIS}^{+}$was confirmed using PCR with primers: POL2_383_f, POL2_2419_f, and conf_5'_TAP_tag.

Yeast strains used to analyze genetic interactions of pol2-16 with pif1 $1 \Delta$ and pol32 24 were constructed as follows: the yWH1096 strain was crossed with YAM. pol32 $:: \mathrm{HPH}$ and diploids were sporulated and tetrads were dissected, pol2-16 (QW670) and pol32::HPH (QW671) segregants were selected. Then QW670 and QW671 were crossed to obtain tetrads, from which pol2-16 (QW676) and pol324: HPH (QW675) were selected. QW675 and QW676 were crossed, yielding a pol2-16 segregant, QW681. Strain QW699 was created by inserting the HPH gene 52 bp after the ORF of POL2 in strain QW681 by standard "ends-out" gene targeting techniques ${ }^{48}$ using the HPH (hygromycin B resistance) module in plasmid pAG32 ${ }^{49}$, amplified by PCR using primers: Oligo_1 and Oligo_2 (Supplementary Data 1) with $50 \mathrm{nt}$ homology to the adjacent regions and $20 \mathrm{nt}$ overlap with the HPH cassette. Strain QW693 resulted from transformation of QW688 with pif1 1 :: KAN cassette, copied from a strain in the yeast knockout collection (GE Healthcare).

Plasmid construction. The integration vector bearing pol2-16 allele was constructed as follows: the pol2-16 allele was amplified using genomic DNA from CWY2201 as template and primers: pol2-16_-364_AvaI and pol2-16_4280_SacI with using KAPA HiFi polymerase (Kapa Biosystems). The restriction digestion of PCR product and integration vector YIplac211 with AvaI and SacI was followed with the DNA purification and subsequent ligation and resulted in the integration vector YIplac211-pol2-16 [pol2-16, URA3].

Doubling time. Five mililiters of YPDA supplemented with additional adenine (90 $\mu \mathrm{g} \mathrm{ml}^{-1}$ ) was inoculated with a single yeast colony (wild type and pol2-4) or a spore colony (pol2-16) and incubated at $23^{\circ} \mathrm{C}$ with rotation (160 r.p.m.). The doubling times $\left(D_{\mathrm{t}}\right)$ of yeast strains were calculated from measurements of the $\mathrm{OD}_{600}$ of exponentially growing yeast cultures over 3.5-day time course. $D_{t}$ were calculated using four to six independent biological replicates of each strain, according to the equation: doubling time $=t / g$, where $g=\left(\log 10\left(N_{t} / N_{0}\right)\right) / 0.3, N_{0}=\mathrm{OD}_{600}$ at start, $N_{t}=\mathrm{OD}_{600}$ at the end, $t=$ time cultured. Presented are the average $D_{t} \pm$ standard deviation.

Flow cytometry. Pre-cultures: $5 \mathrm{ml}$ of YPDA supplemented with additional adenine $\left(90 \mu \mathrm{g} \mathrm{ml}^{-1}\right)$ were inoculated with a single yeast colony (WT or pol2-4 mutant) or a freshly isolated spore colony of pol2-16 mutant, and incubated at $23^{\circ} \mathrm{C}$ with rotation. Pre-cultures were diluted and grown until mid-log phase $\left(\mathrm{OD}_{600}: 0.3-0.8\right)$, then diluted to $\mathrm{OD}_{600}=0.2$, and synchronized with alpha-factor (final concentration in the media $10 \mu \mathrm{g} \mathrm{ml}^{-1}$ ) for $4 \mathrm{~h}$ (after $2 \mathrm{~h}$, an extra portion of alpha-factor was added to final concentration $10 \mu \mathrm{g} \mathrm{ml}^{-1}$ ). Then yeast were collected (3 k.r.p.m., $3 \mathrm{~min}$ ), washed twice with $25 \mathrm{ml}$ of water and $25 \mathrm{ml}$ of YPDA. After the second wash, yeast cells were resuspended in $24 \mathrm{ml}$ of YPDA supplemented with additional adenine $\left(90 \mu \mathrm{g} \mathrm{ml}^{-1}\right)$. Yeast samples were collected every 20 min, mixed with sodium azide (final concentration $0.2 \%$ ), and stored on ice. Yeast cells were collected 3 k.r.p.m., 3 min, RT and fixed in $70 \%$ ethanol, then the DNA was stained with propidium iodide according to the standard protocol. Briefly, yeast cells were resuspended in $50 \mathrm{mM}$ Tris- $\mathrm{HCl}$, $\mathrm{pH} 8.0$ with RNase A (final concentration $1 \mathrm{mg} \mathrm{m}^{-1}$ ) and incubated for $4 \mathrm{~h}$ at $37^{\circ} \mathrm{C}$. Cells were collected (3 k.r.p.m., $3 \mathrm{~min}$ ) and incubated with pepsin (final concentration $5 \mathrm{mg} \mathrm{ml}^{-1}$ ) for 1 $\mathrm{h}$ at $37^{\circ} \mathrm{C}$ and neutralized with $100 \mathrm{mM}$ Tris- $\mathrm{HCl} \mathrm{pH} \mathrm{8.0.} \mathrm{Cells} \mathrm{were} \mathrm{stained} \mathrm{with}$ propidium iodide (final concentration $50 \mu \mathrm{g} \mathrm{ml}^{-1}$ ) overnight at $4{ }^{\circ} \mathrm{C}$, then diluted with $50 \mathrm{mM}$ Tris- $\mathrm{HCl}, \mathrm{pH} 8.0$ and sonicated. The DNA content was analyzed using the LSR II flow cytometer and FACSDiva software (BD Bioscience). Data were collected for 10000 cells per sample. Cells were excited using a $488 \mathrm{~nm}$ argon laser and emission was detected at $585 \mathrm{~nm}$. Additionally, the cell cycle distribution profiles were analyzed using FlowJo software (FlowJo, LLC). Cells were gated on a $\mathrm{PE}$-area versus $\mathrm{PE}$-width to eliminate doublets.

Yeast cells staining. Exponentially growing yeast at $23^{\circ} \mathrm{C}\left(\mathrm{OD}_{600}: \sim 0.5\right)$ in the synthetic complete media supplemented with adenine, was incubated for $1 \mathrm{~h}$ with $1 \mu \mathrm{g} \mathrm{ml}^{-1}$ DAPI. Cells were collected and washed twice with PBS and fixed in $70 \%$ ethanol. Before imaging, yeast were collected and washed twice with water. Cells were imaged using confocal microscope (LSM 710, Carl Zeiss, Inc, oil objective $\times 63$, digital zoom $\times 2$ ).

Immunoblotting. A total of $10 \mathrm{OD}$ units of yeast cells collected at log phase $\left(\mathrm{OD}_{600}: 0.3-0.8\right)$ were resuspended in lysis buffer $(150 \mathrm{mM} \mathrm{NaCl}, 40 \mathrm{mM}$ HEPES and $1 \mathrm{mM}$ DTT) supplemented with protease inhibitors (complete EDTA-free protease inhibitors, Roche), $2 \mathrm{mM}$ PMSF, and lysed by vortexing with glass beads at $4{ }^{\circ} \mathrm{C}$. TAP-tagged polypeptides were detected using peroxidase-anti-peroxidase antibody (PAP, Sigma, P1291) at 1:2000 dilution. As loading control, an antibody against PSTAIR (Sigma, P7962) was used at 1:5000 dilution. Proteins immobilized on membranes were visualized using chemiluminescent substrates for HRP (WesternBright Sirius, advansta), images were taken using G:BOX (SYNGENE). The resulting bands were quantified using Image Quant TL (GE Healthcare Life Sciences). Band intensities measured for six to seven independent isolates were used to calculate the relative level of Pol2p.

HydEn-seq libraries construction. Ribonucleotides were mapped in the genomic DNA using hydrolytic end sequencing technique (HydEn-seq) described by Clausen et al. ${ }^{11}$ Briefly, DNA was isolated from exponentially growing yeast $\left(\mathrm{OD}_{600}: 0.5-1\right)$ with using a kit for genomic DNA isolation from yeast (Epicenter) Libraries for Next Generation Sequencing were prepared with using $1 \mu \mathrm{g}$ of DNA, which was treated with $20 \mathrm{U}$ of restriction enzyme Sbfl-HF (New England Biolabs) at $37^{\circ} \mathrm{C}$ for $1 \mathrm{~h}$. Next DNA was treated under $300 \mathrm{mM} \mathrm{KOH}$ at $55^{\circ} \mathrm{C}$ for $2 \mathrm{~h}$ and precipitated with ethanol. Library were constructed as described earlier ${ }^{11}$. Briefly, DNA was denatured for 3 min at $85^{\circ} \mathrm{C}$, phosphorylated with $10 \mathrm{U}$ of T4 PNK (\#M0236, New England Biolabs) and purified with 1.8 volume of magnetic beads (Magbio). After denaturation for $3 \mathrm{~min}$ at $85^{\circ} \mathrm{C}$, DNA fragments were ligated with adapter ARC140 overnight at $25^{\circ} \mathrm{C}$ using $10 \mathrm{U}$ of T4 RNA ligase (\#M0204, New England Biolabs). This was followed by DNA purification with magnetic beads and the second strand synthesize using $4 \mathrm{U}$ of T7 DNA polymerase (\#M0274, New England Biolabs) and ARC76/77 duplex. After DNA purification with magnetic beads, unique indexes were added to DNA fragments using KAPA HiFi HotStart Ready Mix (\#KK2602, KAPA Biosystems). The library concentrations and sizes were determined using Bioanalyzer. Libraries were pooled and subject for pairedend sequencing with Illumina HiSeq 2500.

Alignment and normalization. HydEn-seq samples were processed as in ref. ${ }^{11}$. Adapter sequence was trimmed from paired-end reads using cutadapt 1.12, discarding pairs where one or both mates were shorter than $15 \mathrm{nt}(-\mathrm{m} \mathrm{15},-\mathrm{q} 10)$. Reads derived from oligos used in library preparation were filtered by aligning mate 1 of each pair to an index containing these sequences, and retaining only those that were not mappable (bowtie 1.2, -v2). Retained pairs were subsequently mapped to the S. cerevisiae W303 assembly, retaining only unique alignments, and trimming a single nucleotide from the $3^{\prime}$ end of each read to allow alignment of $100 \%$ overlapping pairs (bowtie 1.2, -m 1 -v 2 -3 1-best -X1000). Mate 1 of remaining unmapped pairs was then aligned to W303, again retaining only uniquely mappable reads (bowtie 1.2, - $\mathrm{m} 1$-v 2-best). The positions of the $5^{\prime}$ ends of all uniquely mapped mate 1 reads, from both the paired-end and single-end alignments, were shifted upstream by 1 , the implied ribonucleotide location, and bedGraph files containing per-nt counts were generated for each sample using custom scripts.

For the purpose of determining normalization factors, the reads mappable as pairs or mate 1 alone, to multiple locations, were re-aligned with bowtie 1.2 using the same parameters, but omitting -m 1, resulting in a single best alignment for each. BedGraph files were generated as above using all uniquely and non-uniquely mapped reads. From these files, counts of $5^{\prime}$ ends mapping to all Sbfl-HF restriction sites, on both strands, were determined. Normalization factors were then calculated using the method implemented in DESeq ${ }^{50}$ : for each position, the geometric mean of counts for all samples was determined, as well as the ratio of each sample's count to this value. Sites where zero reads were observed in one or more samples were excluded. The median ratio among all Sbfl-HF sites was selected as the normalization factor for each sample. Normalized HydEn-seq counts were subsequently calculated by means of division by these factors.

Identification of origins of replication in $\mathbf{W} \mathbf{3 0 3}$. Origins of replication in the W303 assembly were identified using those annotated to the L03 assembly, previously utilized by Clausen, et al. ${ }^{11}$ To this end, $101 \mathrm{nt}$ sequences centered on each L03 ACS were extracted and aligned to W303 with blat v. 34, using default parameters. Of the 214 sequences examined, 208 were mapped to the same chromosome and strand at full length, five sequences mapped to the same chromosome and strand at $95 \%$ of full length or better, while a single sequence was mapped at $90 \%$. In a single case, the ACS differed by 1 nt between L03 and W303, while for the remaining 213, the ACS was identical. For each alignment, the ACS location in W303 was determined based on its position relative to the L03 sequence's first mapped base.

End-count normalization and meta-analysis. The sum of $5^{\prime}$ ends in bins of $5 \mathrm{bp}$ centered on ACS positions were calculated using BedGraph files and a custom script (heatmap script). These calculations were performed for same and opposite strand independently. Then read counts for 214 origins of replication in each bin 
were sum up and scaled (divided) with the normalization factors calculated based on SbfI-HF restriction sites determined in each sample.

Data availability. The data discussed in this publication have been deposited in NCBI's Gene Expression Omnibus ${ }^{51}$ and are accessible through GEO Series accession number GSE101698. The data that support the findings of this study are available from the corresponding author upon request.

Received: 14 July 2017 Accepted: 1 February 2018

Published online: 27 February 2018

\section{References}

1. Bell, S. P. \& Labib, K. Chromosome duplication in Saccharomyces cerevisiae. Genetics 203, 1027-1067 (2016).

2. Stodola, J. L. \& Burgers, P. M. Mechanism of lagging-strand DNA replication in eukaryotes. Adv. Exp. Med. Biol. 1042, 117-133 (2017).

3. Burgers, P. M. \& Kunkel, T. A. Eukaryotic DNA replication fork. Annu. Rev. Biochem. 86, 417-438 (2017).

4. Lujan, S. A., Williams, J. S. \& Kunkel, T. A. DNA polymerases divide the labor of genome replication. Trends Cell Biol. 26, 640-654 (2016).

5. Miller, T. C. \& Costa, A. The architecture and function of the chromatin replication machinery. Curr. Opin. Struct. Biol. 47, 9-16 (2017).

6. Pursell, Z. F., Isoz, I., Lundstrom, E. B., Johansson, E. \& Kunkel, T. A. Yeast DNA polymerase epsilon participates in leading-strand DNA replication. Science 317, 127-130 (2007).

7. Nick McElhinny, S. A. et al. Abundant ribonucleotide incorporation into DNA by yeast replicative polymerases. Proc. Natl Acad. Sci. USA 107, 4949-4954 (2010).

8. Nick McElhinny, S. A. et al. Genome instability due to ribonucleotide incorporation into DNA. Nat. Chem. Biol. 6, 774-781 (2010).

9. Lujan, S. A. et al. Heterogeneous polymerase fidelity and mismatch repair bias genome variation and composition. Genome Res. 24, 1751-1764 (2014)

10. Williams, J. S., Lujan, S. A. \& Kunkel, T. A. Processing ribonucleotides incorporated during eukaryotic DNA replication. Nat. Rev. Mol. Cell Biol. 17, 350-363 (2016).

11. Clausen, A. R. et al. Tracking replication enzymology in vivo by genome-wide mapping of ribonucleotide incorporation. Nat. Struct. Mol. Biol. 22, 185-191 (2015).

12. Koh, K. D., Balachander, S., Hesselberth, J. R. \& Storici, F. Ribose-seq: global mapping of ribonucleotides embedded in genomic DNA. Nat. Methods 12, 251-257 (2015).

13. Daigaku, Y. et al. A global profile of replicative polymerase usage. Nat. Struct. Mol. Biol. 22, 192-198 (2015).

14. Nick McElhinny, S. A., Gordenin, D. A., Stith, C. M., Burgers, P. M. \& Kunkel, T. A. Division of labor at the eukaryotic replication fork. Mol. Cell 30, 137-144 (2008).

15. Miyabe, I., Kunkel, T. A. \& Carr, A. M. The major roles of DNA polymerases epsilon and delta at the eukaryotic replication fork are evolutionarily conserved. PLoS Genet. 7, e1002407 (2011).

16. Miyabe, I. et al. Polymerase delta replicates both strands after homologous recombination-dependent fork restart. Nat. Struct. Mol. Biol. 22, 932-938 (2015).

17. Yeeles, J. T., Janska, A., Early, A. \& Diffley, J. F. How the eukaryotic replisome achieves rapid and efficient DNA replication. Mol. Cell 65, 105-116 (2017).

18. Tsurimoto, T. \& Stillman, B. Replication factors required for SV40 DNA replication in vitro. II. Switching of DNA polymerase alpha and delta during initiation of leading and lagging strand synthesis. J. Biol. Chem. 266, 1961-1968 (1991).

19. Stillman, B. Reconsidering DNA polymerases at the replication fork in eukaryotes. Mol. Cell 59, 139-141 (2015).

20. Tsurimoto, T. \& Stillman, B. Replication factors required for SV40 DNA replication in vitro. I. DNA structure-specific recognition of a primer-template junction by eukaryotic DNA polymerases and their accessory proteins. J. Biol. Chem. 266, 1950-1960 (1991).

21. Kesti, T., Flick, K., Keranen, S., Syvaoja, J. E. \& Wittenberg, C. DNA polymerase epsilon catalytic domains are dispensable for DNA replication, DNA repair, and cell viability. Mol. Cell 3, 679-685 (1999).

22. Dua, R., Levy, D. L. \& Campbell, J. L. Analysis of the essential functions of the C-terminal protein/protein interaction domain of Saccharomyces cerevisiae pol e and its unexpected ability to support growth in the absence of the DNA polymerase domain. J. Biol. Chem. 274, 22283-22288 (1999).

23. Ohya, T. et al. The DNA polymerase domain of pol(epsilon) is required for rapid, efficient, and highly accurate chromosomal DNA replication, telomere length maintenance, and normal cell senescence in Saccharomyces cerevisiae. J. Biol. Chem. 277, 28099-28108 (2002).

24. Feng, W. \& D'Urso, G. Schizosaccharomyces pombe cells lacking the aminoterminal catalytic domains of DNA polymerase epsilon are viable but require the DNA damage checkpoint control. Mol. Cell. Biol. 21, 4495-4504 (2001).

25. Johnson, R. E., Klassen, R., Prakash, L. \& Prakash, S. A major role of DNA polymerase $\delta$ in replication of both the leading and lagging DNA strands. Mol. Cell 59, 163-175 (2015).

26. Morrison, A., Bell, J. B., Kunkel, T. A. \& Sugino, A. Eukaryotic DNA polymerase amino acid sequence required for $3^{\prime}-5^{\prime}$ exonuclease activity. Proc. Natl Acad. Sci. USA 88, 9473-9477 (1991).

27. Williams, J. S. et al. Proofreading of ribonucleotides inserted into DNA by yeast DNA polymerase varepsilon. DNA Repair. 11, 649-656 (2012).

28. Smith, D. J. \& Whitehouse, I. Intrinsic coupling of lagging-strand synthesis to chromatin assembly. Nature 483, 434-438 (2012).

29. Lydeard, J. R., Jain, S., Yamaguchi, M. \& Haber, J. E. Break-induced replication and telomerase-independent telomere maintenance require Pol32. Nature 448, 820-823 (2007).

30. Wilson, M. A. et al. Pif1 helicase and Poldelta promote recombinationcoupled DNA synthesis via bubble migration. Nature 502, 393-396 (2013).

31. Saini, N. et al. Migrating bubble during break-induced replication drives conservative DNA synthesis. Nature 502, 389-392 (2013).

32. Sengupta, S., van Deursen, F., de Piccoli, G. \& Labib, K. Dpb2 integrates the leading-strand DNA polymerase into the eukaryotic replisome. Curr. Biol. 23, 543-552 (2013).

33. Muramatsu, S., Hirai, K., Tak, Y. S., Kamimura, Y. \& Araki, H. CDKdependent complex formation between replication proteins Dpb11, Sld2, Pol (epsilon\}, and GINS in budding yeast. Genes Dev. 24, 602-612 (2010).

34. Langston, L. D. et al. CMG helicase and DNA polymerase epsilon form a functional 15-subunit holoenzyme for eukaryotic leading-strand DNA replication. Proc. Natl Acad. Sci. USA 111, 15390-15395 (2014).

35. Li, Y. \& Araki, H. Loading and activation of DNA replicative helicases: the key step of initiation of DNA replication. Genes Cells 18, 266-277 (2013).

36. Shinbrot, E. et al. Exonuclease mutations in DNA polymerase epsilon reveal replication strand specific mutation patterns and human origins of replication. Genome Res. 24, 1740-1750 (2014).

37. Haradhvala, N. J. et al. Mutational strand asymmetries in cancer genomes reveal mechanisms of DNA damage and repair. Cell 164, 538-549 (2016).

38. Williams, J. S. et al. Evidence that processing of ribonucleotides in DNA by topoisomerase 1 is leading-strand specific. Nat. Struct. Mol. Biol. 22, 291-297 (2015).

39. Langston, L. D. \& O’Donnell, M. DNA polymerase delta is highly processive with proliferating cell nuclear antigen and undergoes collision release upon completing DNA. J. Biol. Chem. 283, 29522-29531 (2008).

40. Lydeard, J. R. et al. Break-induced replication requires all essential DNA replication factors except those specific for pre-RC assembly. Genes Dev. 24, 1133-1144 (2010)

41. Pinter, S. F., Aubert, S. D. \& Zakian, V. A. The Schizosaccharomyces pombe Pfh1p DNA helicase is essential for the maintenance of nuclear and mitochondrial DNA. Mol. Cell. Biol. 28, 6594-6608 (2008).

42. Yu, C. et al. Strand-specific analysis shows protein binding at replication forks and PCNA unloading from lagging strands when forks stall. Mol. Cell $\mathbf{5 6}$ 551-563 (2014)

43. Zhou, J. C. et al. CMG-Pol epsilon dynamics suggests a mechanism for the establishment of leading-strand synthesis in the eukaryotic replisome. Proc. Natl Acad. Sci. USA 114, 4141-4146 (2017)

44. Georgescu, R. et al. Structure of eukaryotic CMG helicase at a replication fork and implications to replisome architecture and origin initiation. Proc. Natl Acad. Sci. USA 114, E697-E706 (2017).

45. Georgescu, R. E. et al. Mechanism of polymerase collision release from sliding clamps on the lagging strand. EMBO J. 28, 2981-2991 (2009).

46. Schauer, G. D. \& O’Donnell, M. E. Quality control mechanisms exclude incorrect polymerases from the eukaryotic replication fork. Proc. Natl Acad. Sci. USA 114, 675-680 (2017).

47. Chilkova, O. et al. The eukaryotic leading and lagging strand DNA polymerases are loaded onto primer-ends via separate mechanisms but have comparable processivity in the presence of PCNA. Nucleic Acids Res. 35, 6588-6597 (2007)

48. Wach, A., Brachat, A., Pohlmann, R. \& Philippsen, P. New heterologous modules for classical or PCR-based gene disruptions in Saccharomyces cerevisiae. Yeast 10, 1793-1808 (1994).

49. Goldstein, A. L. \& McCusker, J. H. Three new dominant drug resistance cassettes for gene disruption in Saccharomyces cerevisiae. Yeast 15, 1541-1553 (1999).

50. Anders, S. \& Huber, W. Differential expression analysis for sequence count data. Genome Biol. 11, R106 (2010). 
51. Edgar, R., Domrachev, M. \& Lash, A. E. Gene Expression Omnibus: NCBI gene expression and hybridization array data repository. Nucleic Acids Res. 30, 207-210 (2002).

52. Araki, H., Hamatake, R. K., Johnston, L. H. \& Sugino, A. DPB2, the gene encoding DNA polymerase II subunit $\mathrm{B}$, is required for chromosome replication in Saccharomyces cerevisiae. Proc. Natl Acad. Sci. USA 88, 4601-4605 (1991).

53. Araki, H. et al. Cloning DPB3, the gene encoding the third subunit of DNA polymerase II of Saccharomyces cerevisiae. Nucleic Acids Res. 19, 4867-4872 (1991).

54. Hamatake, R. K. et al. Purification and characterization of DNA polymerase II from the yeast Saccharomyces cerevisiae. Identification of the catalytic core and a possible holoenzyme form of the enzyme. J. Biol. Chem. 265, 4072-4083 (1990).

55. Ohya, T., Maki, S., Kawasaki, Y. \& Sugino, A. Structure and function of the fourth subunit (Dpb4p) of DNA polymerase epsilon in Saccharomyces cerevisiae. Nucleic Acids Res. 28, 3846-3852 (2000).

\section{Acknowledgements}

We thank K. Bebenek and D. Gordenin for critical reading of and thoughtful comments on the manuscript. We thank all members of DNA Replication Fidelity Group as well as D. Gordenin, P. Mieczkowski, F. Boellmann, and M. Schellenberg for helpful discussions throughout the work. We are thankful to A. Clausen for original instruction on how to prepare HydEn-seq libraries; members of the Fluorescence Microscopy and Imaging Center/NIEHS for help with yeast imaging; members of the Flow Cytometry Center/ NIEHS for help with Fluorescence-Activated Cell Sorting (FACS) analysis; members of the High Throughput Genomic Sequencing Facility/UNC Chapel Hill for performing the whole-genome sequencing; C. Wittenberg (Scripps Research Institute) for providing pol2-16 mutation source and A. Chabes (Umeå University) for providing the AC402 and AC403 strains. This work was supported by Project Z01 ES065070 to T.A.K. from the Division of Intramural Research of the NIH, NIEHS. Work in the Haber lab was supported by NIH grants GM76020 and P01grant GM105473.

\section{Author contributions}

M.A.G., S.A.L. and T.A.K. designed the experiments. M.A.G. and P.B.C. constructed the experimental system. M.A.G. and P.B.C. carried out the in vivo experiments. M.A.G. constructed HydEn-seq libraries. S.A.L., A.B.B. and M.A.G. performed the HydEn-seq data analysis. Z.-X.Z. suggested restriction enzyme usage to normalize the HydEn-seq data. Q.W. and J.E.H. designed and performed the synthetic genetic interactions experiments. T.A.K., M.A.G. and S.A.L. wrote the manuscript, and all authors edited the manuscript.

\section{Additional information}

Supplementary Information accompanies this paper at https://doi.org/10.1038/s41467 018-03270-4.

Competing interests: The authors declare no competing interests.

Reprints and permission information is available online at http://npg.nature.com/ reprintsandpermissions/

Publisher's note: Springer Nature remains neutral with regard to jurisdictional claims in published maps and institutional affiliations.

(c) (i) Open Access This article is licensed under a Creative Commons Attribution 4.0 International License, which permits use, sharing, adaptation, distribution and reproduction in any medium or format, as long as you give appropriate credit to the original author(s) and the source, provide a link to the Creative Commons license, and indicate if changes were made. The images or other third party material in this article are included in the article's Creative Commons license, unless indicated otherwise in a credit line to the material. If material is not included in the article's Creative Commons license and your intended use is not permitted by statutory regulation or exceeds the permitted use, you will need to obtain permission directly from the copyright holder. To view a copy of this license, visit http://creativecommons.org/ licenses/by/4.0/.

(C) The Author(s) 2018 